\title{
Evaluation of contrast sensitivity measurements after retrobulbar optic neuritis in Multiple Sclerosis
}

\author{
Marta Owidzka • Michal Wilczynski • \\ Wojciech Omulecki
}

Received: 1 August 2013 /Revised: 1 December 2013 / Accepted: 4 February 2014 / Published online: 28 February 2014

(C) The Author(s) 2014. This article is published with open access at Springerlink.com

\begin{abstract}
Background The evaluation of contrast sensitivity is an important additional examination that allows the physician to achieve the full picture of a patient's quality of vision. In lowcontrast conditions, more discrete visual dysfunctions may be revealed, which could be overlooked in high-contrast tests. Methods The examined group consisted of 33 eyes of 27 patients with multiple sclerosis. The study included patients with full or almost full visual acuity, without visual field defects or any other ophthalmic condition, and who had never undergone any ocular surgery or trauma. The reference group consisted of 49 eyes of 37 patients. This group included healthy subjects with full visual acuity. Contrast sensitivity was examined with a Functional Vision Analyzer ${ }^{\mathrm{TM}}$ device in photopic conditions (with and without glare) and in mesopic conditions (with and without glare).

Results In patients with multiple sclerosis who had experienced optic neuritis, contrast sensitivity was found to be significantly reduced in all spatial frequencies in both mesopic and photopic conditions (with and without glare).

Conclusions Contrast sensitivity in patients with multiple sclerosis who have also had optic neuritis is significantly reduced. This may explain patients' complaints regarding their quality of vision, despite good visual acuity. Contrastometry is a useful basis for further examination, providing additional information regarding a patient's quality of vision.
\end{abstract}

Keywords Contrast sensitivity · Multiple sclerosis ·

Retrobulbar optic neuritis

M. Owidzka $(\bowtie) \cdot$ M. Wilczynski $\cdot$ W. Omulecki

Department of Ophthalmology, Medical University of Lodz, Medical

University Barlicki Hospital No.1, Kopcinskiego str. 22,

90-153 Lodz, Poland

e-mail: mowidzka@gmail.com

\section{Introduction}

The evaluation of contrast sensitivity is an important examination that allows us to recognize many visual disfunctions in their early stages and to achieve the full picture of a patient's quality of vision [1-3]. In various diseases, the ability to discern low-contrast objects may be decreased, while the ability to recognize high-contrast objects may be unaffected.

During a routine ophthalmic examination, the evaluation of visual acuity with Snellen or logMAR charts is one of basic tests performed to examine visual functions. However, it is necessary to be aware of the limitations of examinations using these charts, as they are designed with high-contrast optotypes [4-7].

In low-contrast conditions, more discrete visual dysfunctions may be revealed that could be otherwise overlooked in high-contrast tests $[5,8-15]$. The evaluation of contrast sensitivity is a subjective measurement of visual potential and provides information about the quality of a patient's vision $[3$, $15]$.

In most life situations, we have contact with objects of lower contrast than those on Snellen charts. For this reason, the evaluation of contrast sensitivity allows us to understand why among people with similar visual acuity, some patients complain of decreased vision and visual functioning in everyday life [13, 14, 16-19].

Contrastometry is used mainly to diagnose patients with ophthalmic diseases or patients who have undergone refractive or cataract surgery, but it is also useful in the examination of patients with neurological diseases, general medical conditions, neurotoxical disorders, and of some healthy people, in whom it is important to evaluate visual quality (e.g., pilots, drivers, etc.).

Demyelinization in the course of multiple sclerosis (MS) is one of the most frequent causes of optic neuritis. It is estimated that about $15-20 \%$ of patients with multiple sclerosis are 
diagnosed with optic neuritis in the course of the disease [1, 20]. There are articles suggesting that this proportion is much higher and may be as high as $50 \%[1,21,22]$. The main symptoms include: subacute, unilateral, and decreased visual acuity, which may be accompanied by dyschromatopsy, decreased contrast sensitivity, phosphenes (positive visual phenomena) seen as white or colorful flashes, as well as ocular pain or pain around the eyeball, which increases with eye movement and afferent pupillary defect. In addition, visual field defects or, more frequently, generalized decreased retinal sensitivity may be present. Decreased contrast sensitivity is present in about $60-80 \%$ of patients with MS; nevertheless, some of them have normal visual acuity [23-25].

The purpose of our study was to evaluate contrast sensitivity in patients with multiple sclerosis who had also suffered from optic neuritis, as well as to estimate the clinical usefulness of the Functional Vision Analyzer ${ }^{\mathrm{TM}}$ in the diagnostics of these patients.

\section{Methods}

The examined group consisted of 33 eyes of 27 patients (10 men and 17 women), aged from 19 to 48 years old (mean 32 years), diagnosed with multiple sclerosis. The study included only patients with good best-corrected visual acuity $(\mathrm{BCVA}$ ), ranging from 0.7 to 1.0 (mean $\mathrm{BCVA}=0.97$ ) without visual field defects or any other ophthalmic condition. Other inclusion criteria were: the lack of any visual symptoms, no ocular disease, no previous ocular surgery or trauma, as well as the absence of any general medical conditions. In cases when the patient experienced optic neuritis unilaterally, only one eye was included in the study. The duration of multiple sclerosis ranged from 1 to 16 years. The time between the episode of optic neuritis and our contrast sensitivity measurement exceeded 6 months in all cases.

The reference group consisted of 49 eyes of 37 patients ( 16 women and 21 men), aged from 20 to 81 years old (mean 43 years). This group included healthy subjects, with full visual acuity, who had never been diagnosed with any ophthalmic disease.

The analyzed data were gathered prospectively from a nonrandomized consecutive series of patients. For all study protocols, we followed all tenets of the Declaration of Helsinki. All patients gave an informed consent to participate in the study. Before the study, the Ethics Committee approval was obtained (number RNN/53/11/KE).

All patients had a full ophthalmic examination before contrastometry was performed. Best-corrected visual acuity was examined with standard Snellen charts (numeral optotypes). Slit-lamp examination of the anterior segment and the eye fundus was performed. Intraocular pressure was measured with an applanation tonometer. In addition, retinal nerve fiber layer (RNFL) thickness was measured using optical coherence tomography (OCT).

In all patients, contrast sensitivity was examined with a Functional Vision Analyzer ${ }^{\mathrm{TM}}$ (Stereo Optical Co., Inc.) device in photopic conditions (with and without glare) and in mesopic conditions (with and without glare). In all patients, contrastometry was performed in the same lightning conditions, with a "distance examination strategy". Patients who had a refractive error were examined with appropriate correction.

We also evaluated vision (with and without glare, 1 lux) and day vision (with and without glare, 10 lux) in each patient, for each eye separately. Contrast sensitivity was measured for the following spatial frequencies: $1.5,3,6,12$, and $18 \mathrm{cpd}$ (cycles per degree).

\section{Results}

The results of contrast sensitivity measurements show that there is a significant difference in the mean contrast sensitivity between group of patients after optic neuritis in multiple sclerosis and healthy subjects (Table 1). Furthermore, in patients with multiple sclerosis, contrast sensitivity is significantly reduced in all spatial frequencies, both in photopic conditions (with and without glare) and in mesopic conditions (with and without glare) (Table 2).

Our study also confirms that a reduction of contrast sensitivity is observable in all spatial frequencies-low, medium, and high.

The results of OCT measurements in a group of patients with multiple sclerosis show that retinal nerve fiber layer thickness (RNFL) was between 56 and $122 \mu \mathrm{m}$ (mean 98.3; standard deviation 16.2). All patients with MS, except one, had RNFL thickness above $75 \mu \mathrm{m}$. RNFL thickness in a group of healthy control subjects was from 78 to $126 \mu \mathrm{m}$ (mean 100.9; standard deviation 14). There were no statistically significant differences between mean RNFL thickness in both groups.

\section{Discussion}

Decreased contrast sensitivity is noticeable in low, intermediate, and high spatial frequencies in patients with an acute inflammatory phase, in patients after neuritis, and in patients with multiple sclerosis without a history of optic neuritis [23]. In patients who haven't had optic neuritis in the course of multiple sclerosis, contrast sensitivity was decreased in intermediate and high spatial frequencies (12-18 cpd) [26].

In patients with optic neuritis, an increase in visual acuity can be observed after 2-4 weeks, to the level of 0.6 or better. Nevertheless, color perception and contrast sensitivity are 
Table 1 Comparison of contrast sensitivity measurements in various conditions, in patients after optic neuritis with the control group

$M S$ multiple sclerosis, $x$ mean, $S D$ standard deviation

Table 2 Statistics of contrast sensitivity measurements, in daylight/night conditions, with and without glare, in patients after optic neuritis

min minimum, $\max$ maximum, $x$ mean $M e$ median, $S D$ standard deviation, $v$ variance

\begin{tabular}{|c|c|c|c|c|c|c|c|}
\hline \multirow[t]{3}{*}{ Examination } & \multirow{3}{*}{$\begin{array}{l}\text { Spatial } \\
\text { frequency }\end{array}$} & \multicolumn{4}{|c|}{ Group } & \multirow[t]{3}{*}{$\mathrm{Z}$ test } & \multirow[t]{3}{*}{ Significance $p$} \\
\hline & & \multicolumn{2}{|c|}{ MS with optic neuritis } & \multicolumn{2}{|c|}{ Control group } & & \\
\hline & & $\mathrm{x}$ & SD & $\mathrm{x}$ & SD & & \\
\hline \multirow{5}{*}{$\begin{array}{l}\text { Night testing without } \\
\text { glare }\end{array}$} & 1.5 & 31.9 & 12.1 & 56.0 & 25.1 & 4.515 & $\mathrm{p}<0.001$ \\
\hline & 3 & 70.4 & 32.9 & 104.3 & 34.7 & 3.990 & $\mathrm{p}<0.001$ \\
\hline & 6 & 34.3 & 23.4 & 69.3 & 33.5 & 4.577 & $\mathrm{p}<0.001$ \\
\hline & 12 & 8.45 & 7.52 & 21.4 & 16.4 & 4.174 & $\mathrm{p}<0.001$ \\
\hline & 18 & 0.91 & 2.50 & 4.76 & 4.48 & 4.005 & $\mathrm{p}<0.001$ \\
\hline \multirow[t]{5}{*}{ Night testing with glare } & 1.5 & 39.9 & 19.2 & 65.7 & 23.9 & 4.477 & $\mathrm{p}<0.001$ \\
\hline & 3 & 72.1 & 34.2 & 109.0 & 31.2 & 4.298 & $\mathrm{p}<0.001$ \\
\hline & 6 & 35.9 & 27.7 & 68.4 & 30.8 & 4.709 & $\mathrm{p}<0.001$ \\
\hline & 12 & 9.79 & 8.34 & 20.9 & 15.5 & 3.744 & $\mathrm{p}<0.001$ \\
\hline & 18 & 0.61 & 1.97 & 4.82 & 5.13 & 4.094 & $\mathrm{p}<0.001$ \\
\hline \multirow[t]{5}{*}{ Day testing without glare } & 1.5 & 28.6 & 12.7 & 50.4 & 19.3 & 5.238 & $\mathrm{p}<0.001$ \\
\hline & 3 & 79.7 & 33.4 & 117.1 & 27.9 & 4.553 & $\mathrm{p}<0.001$ \\
\hline & 6 & 52.6 & 30.5 & 108.0 & 38.7 & 5.569 & $\mathrm{p}<0.001$ \\
\hline & 12 & 22.1 & 14.4 & 41.3 & 24.4 & 3.995 & $\mathrm{p}<0.001$ \\
\hline & 18 & 6.42 & 5.40 & 16.2 & 11.5 & 4.189 & $\mathrm{p}<0.001$ \\
\hline \multirow[t]{5}{*}{ Day testing with glare } & 1.5 & 40.2 & 15.8 & 64.3 & 23.6 & 4.392 & $\mathrm{p}<0.001$ \\
\hline & 3 & 84.9 & 30.9 & 127.4 & 26.1 & 5.064 & $\mathrm{p}<0.001$ \\
\hline & 6 & 59.9 & 39.1 & 112.3 & 37.9 & 5.201 & $\mathrm{p}<0.001$ \\
\hline & 12 & 25.9 & 20.0 & 47.0 & 25.8 & 4.028 & $\mathrm{p}<0.001$ \\
\hline & 18 & 7.15 & 6.01 & 17.5 & 12.5 & 3.971 & $\mathrm{p}<0.001$ \\
\hline
\end{tabular}

\begin{tabular}{|c|c|c|c|c|c|c|c|}
\hline \multirow[t]{2}{*}{ Examination } & \multirow{2}{*}{$\begin{array}{l}\text { Spatial } \\
\text { frequency }\end{array}$} & \multicolumn{6}{|c|}{ Calculated contrast parameters } \\
\hline & & $\min$ & $\max$ & $\mathrm{x}$ & $\mathrm{Me}$ & SD & $\mathrm{v}(\%)$ \\
\hline \multirow[t]{5}{*}{ Night testing without glare } & 1.5 & 13 & 50 & 31.9 & 36 & 12.1 & 37.8 \\
\hline & 3 & 10 & 160 & 70.4 & 57 & 32.9 & 46.7 \\
\hline & 6 & 0 & 90 & 34.3 & 33 & 23.4 & 68.4 \\
\hline & 12 & 0 & 22 & 8.45 & 8 & 7.52 & 88.9 \\
\hline & 18 & 0 & 12 & 0.91 & 0 & 2.50 & 275.5 \\
\hline \multirow[t]{5}{*}{ Night testing with glare } & 1.5 & 18 & 71 & 39.9 & 36 & 19.2 & 48.1 \\
\hline & 3 & 10 & 160 & 72.1 & 57 & 34.2 & 47.4 \\
\hline & 6 & 0 & 128 & 35.9 & 33 & 27.7 & 77.0 \\
\hline & 12 & 0 & 30 & 9.79 & 11 & 8.34 & 85.2 \\
\hline & 18 & 0 & 8 & 0.61 & 0 & 1.97 & 324.6 \\
\hline \multirow[t]{5}{*}{ Day testing without glare } & 1.5 & 13 & 71 & 28.6 & 25 & 12.7 & 44.2 \\
\hline & 3 & 29 & 160 & 79.7 & 80 & 33.4 & 41.9 \\
\hline & 6 & 0 & 128 & 52.6 & 45 & 30.5 & 57.9 \\
\hline & 12 & 0 & 60 & 22.1 & 22 & 14.4 & 65.3 \\
\hline & 18 & 0 & 17 & 6.42 & 8 & 5.40 & 84.0 \\
\hline \multirow[t]{5}{*}{ Day testing with glare } & 1.5 & 18 & 71 & 40.2 & 36 & 15.8 & 39.3 \\
\hline & 3 & 29 & 160 & 84.9 & 80 & 30.9 & 36.4 \\
\hline & 6 & 0 & 180 & 59.9 & 45 & 39.1 & 65.3 \\
\hline & 12 & 0 & 85 & 25.9 & 22 & 20.0 & 77.3 \\
\hline & 18 & 0 & 17 & 7.15 & 8 & 6.01 & 84.0 \\
\hline
\end{tabular}


reduced. In about $10 \%$ of patients, chronic optic neuritis develops, with no remissions and with a constant and gradual decrease of visual acuity $[1,20,27-31]$.

Degeneration of the optic nerve axons causes the disturbance of retinal nerve fiber layer thickness, which can be documented with OCT. What's more, OCT allows an estimate of the relationship between RNFL thickness and visual function after optic neuritis. It is thought that the first changes in RNFL thickness can be seen in the temporal quadrant, as early as 2 months after the inflammatory episode. The decrease in RNFL thickness in eyes after optic neuritis is visible for up to 24 months; during the first 6 months the changes increase, and later on, they stabilize [32].

Regression analysis helped to determine the minimal RNFL thickness (amounting to $75 \mu \mathrm{m}$ ) that allowed for the recovery of vision after optic neuritis [32,33]. Retinal nerve fiber thickness was found to correlate with visual acuity, contrast sensitivity, color vision, and visual field defects [20].

There was a significant decrease of RNFL thickness in eyes after optic neuritis caused by multiple sclerosis, in comparison with the fellow eye and with healthy subjects. In addition, an RNFL below $75 \mu \mathrm{m}$ was significantly correlated with visual field defects. It has been observed that despite the fact that visual acuity returned to normal after optic neuritis, patients still complain of subjective decreased vision, which may result from decreased contrast sensitivity or visual field defects $[21,31,34]$.

There are few characteristic types of changes in contrast sensitivity in patients who had optic neuritis in multiple sclerosis: (1) decreased contrast sensitivity in all spatial frequencies; (2) decreased contrast sensitivity in intermediate and high spatial frequencies; (3) decreased contrast sensitivity in only intermediate spatial frequencies; (4) decreased contrast sensitivity in only low spatial frequencies; and (5) unchanged contrast sensitivity [31]. The last two types mentioned are the least frequently encountered.

In examinations evaluating color vision and contrast sensitivity in patients with multiple sclerosis, it has been observed that defects may be selective or generalized. Dain et al. [29] proved the existence of two outcomes among patients after optic neuritis. The first group had a selective defect of color vision in the red-green axis, as well as selective impairment of contrast sensitivity in high spatial frequencies. The second group had a generalized defect of color vision, concerning both the red-green axis and blue-yellow axis, as well as a generalized impairment of contrast sensitivity irrespective of spatial frequency. The authors conclude that this phenomenon may result from the demyelinative damage to the optic nerve, which may be either selective and include mainly small axons, or that it may be generalized and include both small and large axons.

Rekas et al. [35] draw similar conclusions regarding selective and non-selective damage to the optic nerve in patients with multiple sclerosis. They found that one group of patients had decreased contrast sensitivity at $18 \mathrm{cpd}$, as well as defective blue-color perception, whereas the second group of patients had decreased contrast sensitivity at $12 \mathrm{cpd}$, as well as defective red-color perception. In the third (and the largest) group of patients, there was a generalized (nonselective) defect in color perception and generalized decreased contrast sensitivity, with a predilection for higher spatial frequencies.

The results of our study are consistent with the results of previously published articles. However, we have performed a full evaluation of contrast sensitivity in photopic conditions (with and without glare), as well as in mesopic conditions (with and without glare) in patients with MS, which revealed a non-selective significant reduction of contrast sensitivity. Furthermore, this study emphasizes the important role of contrastometry in patients with MS, as a method that can identify the deficits of visual function that otherwise go undetected by a simple visual acuity measurement.

\section{Conclusions}

Contrast sensitivity in patients with multiple sclerosis who also suffer from optic neuritis is significantly reduced in all spatial frequencies, both in photopic conditions (with and without glare) and in mesopic conditions (with and without glare). This may explain patients' complaints regarding their quality of vision, despite having good visual acuity.

Contrastometry is a useful basis for examination in this context, providing additional information regarding the patient's quality of vision.

Acknowledgments The study was supported by grant number 502-03/ 1-007-01/502-14-034 from the Medical University of Lodz, Poland, to Dr. Owidzka.

Conflict of interest The authors confirm that they do not have any commercial or proprietary interests in any product or company mentioned. The authors declare that they have no conflicts of interest to report.

Open Access This article is distributed under the terms of the Creative Commons Attribution License which permits any use, distribution, and reproduction in any medium, provided the original author(s) and the source are credited.

\section{References}

1. Kanski J (2007) Clinical ophthalmology. A systematic approach, Elsevier, Wroclaw

2. Bosse JC (1985) Clinical Use of Contrast Sensitivity Test Plates for Amblyopia. J Optom Vis Dev 26:24-27

3. Thayaparan K, Crossland MD, Rubin GS (2007) Clinical assessment of two new contrast sensitivity charts. Br J Ophthalmol 91:749-752 
4. Lederer P, Bosse J (1992) Clinical use of contrast sensitivity evaluation for general practice of optometry. Practical Optometry 3:34-48

5. Giersch A, Speeg-Schatz C, Monique Tondre M, Gottenkiene S (2006) Impairment of contrast sensitivity in long-term lorazepam users. Psychopharmacology 186:594-600

6. Wood JM, Owens DA (2005) Standard measures of visual acuity do not predict drivers' recognition performance under day or night conditions. Optom Vis Sci 82:698-705

7. Onal S, Yenice O, Cakir S, Temel A (2008) FACT contrast sensitivity as a diagnostic tool in glaucoma. IntOphthalmol 28:407-412

8. Regan D, Neima D (1983) Low-contrast letter charts as a test of visual function. Ophthalmology 90:1192-1200

9. Abrahamsson M, Sjöstrand J (1986) Impairment of contrast sensitivity function (CSF) as a measure of disability glare. Invest Ophthalmol Vis Sci 27:1131-1136

10. Jindra L, Zemon V (1989) Contrast sensitivity testing: A more complete assessment of vision. J Cataract Refract Surg 15:141-148

11. Chua BE, Mitchell P, Cumming RG (2004) Effects of cataract type and location on visual function: the Blue Mountains Eye Study. Eye 18:765-772

12. Rydberg A, Han Y, Lennerstrand G (1997) A comparison between different contrast sensitivity tests in the detection of amblyopia. Strabismus 5:167-184

13. Stifter E, Sacu S, Thaler A, Weghaupt H (2006) Contrast acuity in cataracts of different morphology and association to self-reported visual function. Invest Ophthalmol Vis Sci 47:5412-5422

14. Alexander KR, Barnes CS, Fishman GA, Pokorny J, Smith VC (2004) Contrast sensitivity deficits in inferred magnocellular and parvocellular pathways in retinitis pigmentosa. Invest Ophthalmol Vis Sci 45:4510-4519

15. Hong YT, Kim SW, Kim EK, Kim TI (2010) Contrast sensitivity measurement with 2 contrast sensitivity tests in normal eyes and eyes with cataract. J Cataract Refract Surg 36:547-552

16. West SK, Rubin GS, Broman AT, Muñoz B, Bandeen-Roche K, Turano K (2002) How does visual impairment affect performance on tasks of everyday life? Arch Ophthalmol 120: 774-780

17. Leat SJ, Woo GC (1997) The validity of current clinical tests of contrast sensitivity and their ability to predict reading speed in low vision. Eye 11:893-899

18. Shandiz JH, Derakhshan A, Daneshyar A, Azimi A, Moghaddam HO, Yekta AA, Yazdi SHH, Esmaily H (2011) Effect of cataract type and severity on visual acuity and contrast sensitivity. J Ophthalmic Vis Res 6:26-31

19. Superstein R, Boyaner D, Overbury O (1999) Functional complaints, visual acuity, spatial contrast sensitivity, and glare disability in preoperative and postoperative cataract patients. J Cataract Refract Surg 25:575-581
20. Kallenbach K, Frederiksen J (2007) Optical coherence tomography in optic neuritis and multiple sclerosis: a review. Eur J Neurol 14: 841-849

21. Merle H, Olindo S, Donnio A, Richer R, Smadja D, Cabre P (2011) Anatomic and functional correlation of frequency-doubling technology perimetry (FDTP) in multiple sclerosis. Int Ophthalmol 31:263-270

22. Cheng H, Laron M, Schiffman JS, Tang RA, Frishman LJ (2007) The relationship between visual field and Retinal Nerve Fiber Layer measurements in patients with Multiple Sclerosis. Invest Ophthalmol Vis Sci 48:5798-5805

23. Sanders EA, Volkers AC, van der Poel JC, van Lith GH (1986) Estimation of visual function after optic neuritis: a comparison of clinical tests. Br J Ophthalmol 70:918-924

24. Della Sala S, Comi G, Martinelli V, Somazzi L, Wilkins AJ (1987) The rapid assessment of visual dysfunction in multiple sclerosis. J Neurol Neurosurg Psychiatry 50:840-846

25. Wender M (2007) Value of Pelli-Robson contrast sensitivity chart for evaluation of visual system in multiple sclerosis patients. Neurol Neuroch Pol 41:141-143

26. Sisto D, Trojano M, Vetrugno M, Trabucco T, Iliceto G, Sborgia C (2005) Subclinical visual involvement in multiple sclerosis: a study by MRI, VEPs, frequency-doubling perimetry, standard perimetry, and contrast sensitivity. Invest Ophthalmol Vis Sci 46:1264-1268

27. Pane A, Burdon MA, Miller NR. (2006) The Neuro-ophthalmology survival guide, Elsevier

28. Beck RW, Ruchman MC, Savino PJ, Schatz NJ (1984) Contrast sensitivity measurements in acute and resolved optic neuritis. Br J Ophthalmol 68:756-759

29. Dain SJ, Rammohan KW, Benes SC, King-Smith PE (1990) Chromatic, spatial, and temporal losses of sensitivity in multiple sclerosis. Invest Ophthalmol Vis Sci 31:548-558

30. Schneck ME, Haegerstrom-Portnoy G (1997) Color vision defect type and spatial vision in the optic neuritis treatment trial. Invest Ophthalmol Vis Sci 38:2278-2289

31. Zimmern RL, Campbell FW, Wilkinson IM (1979) Subtle disturbances of vision after optic neuritis elicited by studying contrast sensitivity. J Neurol Neurosurg Psychiatry 42:407-412

32. Kujawa-Hadrys M, Wilczynski M (2011) Importance of the retinal nerve fiber layer examination in various ocular diseases. Okulistyka 3:69-74

33. Costello F, Hodge W, Pan YI, Eggenberger E, Coupland S, Kardon RH (2008) Tracking retinal nerve fiber layer loss after optic neuritis: a prospective study using optical coherence tomography. Mult Scler 14:893-905

34. Steel DH, Waldock A (1998) Measurement of the retinal nerve fibre layer with scanning laser polarimetry in patients with previous demyelinating optic neuritis. J Neurol Neurosurg Psychiatry 64:505-509

35. Rekas M, Pawlik R, Wierzbowska J (2004) The optic nerve damage in multiple sclerosis patients. Klin Oczna 106:512-514 